\title{
Síndrome do disco adjacente à fusão (Síndrome de Transição) na coluna cervical: resultados segundo critérios clínicos e radiológicos
}

\author{
Adjacent segment disease (Transitional Syndrome) in cervical spine: \\ clinical and radiological results
} Síndrome del disco adyacente a la fusión (Síndrome de Transición) en la columna cervical

\author{
Alexandre Meluzzi ${ }^{1}$ \\ Mário Augusto Taricco² \\ Vinícius Monteiro de Paula Guirado' \\ Mário Rubem Pena Dias' \\ Gilberto Nakaguawa' \\ Manoel Jacobsen Teixeira ${ }^{3}$
}

\section{RESUMO}

Objetivo: o objetivo deste trabalho foi identificar a ocorrência da síndrome do disco adjacente secundária à fusão intervertebral, no segmento cervical, e os fatores prognósticos associados. Métodos: foram analisados os prontuários e exames de imagem de 209 indivíduos submetidos ao tratamento cirúrgico da doença degenerativa cervical, sendo 169 casos por via anterior e em 40 por via posterior, no HC-FMUSP, no período de março de 1993 a janeiro de 2007. A avaliação clínica foi quantificada pela escala da Japanese Orthopaedic Association (JOA), com média de segmento de 80 meses. A análise radiológica foi baseada nos critérios de instabilidade de White e Panjabi em todos os casos. A degeneração discal foi mensurada segundo a escala de Kellgren antes e após a cirurgia. Resultados: no total,

\begin{abstract}
Objective: the objective of this study was to identify the occurrence of adjacent disc syndrome secondary to intervertebral fusion in the cervical segment, and the predictors of outcome. Methods: the medical records and imaging studies of 209 patients undergoing surgical treatment of cervical degenerative disease, with 169 cases treated through anterior approach and 40 cases through the posterior approach, at the HC-FMUSP from March 1993 to January 2007. Clinical evaluation was quantified by the scale of Japanese Orthopaedic Association (JOA), with an average follow-up of 80 months. The radiological analysis was based on the criteria of instability by White and Panjabi in all cases. The degeneration was measured by the scale of Kellgren before and
\end{abstract}

\section{RESUMEN}

Objetivo: el objetivo de este trabajo es identificar la ocurrencia del síndrome del disco adyacente secundario a la fusión intervertebral, en el segmento cervical, y los factores pronósticos asociados. Métodos: Fueron analizados las historias clínicas y exámenes de imagen, de 209 individuo sometidos al tratamiento quirúrgico de la enfermedad degenerativa cervical, por vía anterior en 169 casos; $y$ en 40 por vía posterior, en el HC-FMUSP, en el periodo de marzo de 1993 a enero de 2007. La evaluación clínica fue cuantificada por la escala de Japanese Ortopaedic Association (JOA), con un promedio de segmento de 80 meses. El análisis radiológico fue fundamentado en los criterios de inestabilidad de White \& Panjabi, en todos los casos. La degeneración discal fue medida según la escala de Kellgren, antes y después de la

\footnotetext{
Trabalho realizado no Grupo de Cirurgia da Coluna Vertebral, Subdivisão de Neurocirurgia, Departamento de Neurologia do Hospital das Clínicas da Faculdade de Medicina da Universidade de São Paulo - USP - São Paulo (SP), Brasil.

Médicos Neurocirurgiões; Pesquisadores; Assistentes do Grupo de Cirurgia da Coluna Vertebral, Hospital das Clínicas da Faculdade de Medicina da Universidade de São Paulo - USP - São Paulo (SP), Brasil.

2 Livre-docente; Professor do Departamento de Neurologia da Faculdade de Medicina da Universidade de São Paulo, Chefe do Grupo de Cirurgia da Coluna Vertebral do Hospital das Clínicas da Faculdade de Medicina da Universidade de São Paulo - USP - São Paulo (SP), Brasil.

${ }^{3}$ Professor Titular de Neurocirurgia da Faculdade de Medicina da Universidade de São Paulo - USP - São Paulo (SP), Brasil. 
diagnosticou-se a síndrome do disco adjacente em 30 casos $(17,75 \%)$, com média de intervalo de tempo de incidência de 28,5 meses após a fusão ou 2,9 casos/ano. Houve um aumento das alterações degenerativas nos exames radiológicos em níveis adjacentes à fusão por via anterior em $41,42 \%$ e em $52,5 \%$ por via posterior sem fusão, avaliados pela escala de Kellgren, com taxa de degeneração de $6,7 \%$ ao ano, sem correlação com alterações clínicas. Conclusões: alterações no alinhamento fisiológico sagital da coluna e a sua gravidade predispõem ao desenvolvimento da síndrome. Não foi observada incidência da síndrome em casos de pseudoartrose ou utilização de metacrilato, portanto onde não ocorreu a fusão. A utilização de descompressão e fusão, por via anterior, está associada à possibilidade de desenvolvimento de um novo quadro de mielorradiculopatia no período pós-operatório. Fusão em um nível que envolva a quinta ou sexta vértebras cervicais, fixação com placas e pré-existência da evidência radiográfica de degeneração de disco adjacente mostraram-se como os grandes fatores de risco para o seu surgimento.

\section{DESCRITORES: Doenças da} coluna vertebral; Fusão vertebral; Síndrome; Disco intervertebral after surgery. Results: overall, the syndrome of adjacent disc was diagnosed in 30 cases (17.75\%), with a mean time interval of incidence of 28.5 months after the merger, or 2.9 cases per year. There was an increase in degenerative changes in X-rays at levels adjacent to anterior fusion in $41.42 \%$ and $52.5 \%$ for the posterior without fusion, evaluated by the scale of Kellgren, the degeneration rate of $6.7 \%$ per annum, with no correlation with clinical changes. Conclusions: changes in physiological sagittal alignment of the spine and their severity predispose to the development of the syndrome. There was no incidence of the syndrome in cases of pseudoarthrosis or methacrylate, therefore, where there was a merger. The use of decompression and fusion, anterior, is associated with the possibility of developing a new framework for myeloradiculopathy in the postoperative period. Merger at a level that involve the fifth or sixth cervical vertebra, fixation with plates and pre-existing radiographic evidence of disc degeneration adjacent were factors of high risk for its emergence. cirugía. Resultados: en el total, fueron diagnosticados 30 caos de síndrome del disco adyacente (17.75\%), con un promedio de intervalo de tiempo de incidencia de 28.5 meses después de la fusión, o 2.9 casos/año. Hubo un aumento de las alteraciones degenerativas en los exámenes radiológicos, en niveles adyacentes a la fusión por vía anterior en $41.42 \%$, y en $52.5 \%$ por vía posterior sin fusión, evaluados por la escala de Kellgren, con tasa de degeneración de $6.7 \%$ al año, sin correlación con alteraciones clínicas. Conclusiones: Alteraciones en el alineamiento fisiológico sagital de la columna y el agravamiento de éstas, predisponen al desarrollo del sindrome. No fueron observados casos del síndrome en casos de pseudoartrosis o utilización de metacrilato, por lo tanto donde no hubo la fusión. La utilización de descompresión y fusión, por vía anterior están asociadas a la posibilidad del desarrollo de un nuevo cuadro de mielorradiculopatía en el periodo postoperatorio. Una fusión en un nivel incluyendo la quinta y sexta vértebra cervical, fijación con placas y preexistiendo la evidencia radiológica de degeneración de disco adyacente, se mostraron como los grandes factores de riesgo para el seguimiento.

DESCRIPTORES: Enfermedades de la columna vertebral; Fusión vertebral; Síndrome; Disco intervertebral

\section{INTRODUÇÃO}

Inicialmente, a doença degenerativa da coluna cervical era tratada pela via posterior, sem a prática da fusão, com sucesso para a radiculopatia cervical pelo acesso posterolateral conforme publicações de Spurling e Scoville ${ }^{1}$ de 1944. $\mathrm{O}$ acesso anterior tem sido amplamente utilizado para o tratamento da espondilopatia cervical sintomática desde as duas primeiras descrições técnicas em 1958, nos procedimentos de Simth-Robinson (1955) e Cloward (1958) ${ }^{2-4}$, cirurgias que envolviam a descompressão e fusão intersomática. A descompressão e a fusão, nas últimas décadas, tornaram-se os tratamentos de escolha para muitas patologias da coluna vertebral, como escoliose juvenil e estenose lombar e cervical. Na coluna cervical, a descompressão seguida de fusão tem mostrado resultados muito satisfa- tórios no alívio da dor radicular, na estabilização e até na melhora do quadro neurológico na mielopatia com taxas de sucesso muito satisfatórias, em algumas séries em torno de $90 \% 0^{5-8}$.

A despeito desse grande sucesso imediato em casos de estenose, complicações tardias passaram a ser relatadas com cada vez mais frequência, como as alterações dos discos adjacentes à fusão, que podem levar ao desenvolvimento de novos quadros de radiculopatia ou mielopatia precoce ou tardiamente ${ }^{9,10}$. Contudo, nas últimas duas décadas, a fusão tem sido praticada em indivíduos cada vez mais jovens e as indicações têm sido expandidas, incluindo quadros de dor cervical mecânica ou discogênica, sem quadros de dor radicular ou comprometimento medular, com taxas de sucesso variáveis, ao passo que descrições 
de complicações em discos adjacentes têm aumentado progressivamente $^{11,12}$.

A síndrome do disco adjacente, definida como uma deterioração anatômica, funcional, gradual e anormal de um ou ambos os discos intervertebrais adjacentes a um segmento, ou segmentos, de fusão cirúrgica intervertebral, associada ao surgimento de um novo quadro de radiculopatia ou mielopatia, tem sido bem documentada na literatura recente, porém ainda existe controvérsia sobre quando uma degeneração de disco adjacente radiográfica passa e ter relevância clínica.

Alguns estudos têm tornado evidente que a manutenção do alinhamento e do balanço sagital do complexo espinhal tem implicações clínicas na prevenção e no tratamento da degeneração dos discos adjacentes ${ }^{13,14}$. Entretanto, a literatura não traz, de forma clara, se estas degenerações são subsequentes ao processo de fusão ou em função do processo natural de progressão da doença degenerativa da coluna cervical ${ }^{13-16}$.

É teoricamente determinada por uma sobrecarga mecânica imposta pela imobilização dos segmentos fundidos, transferindo energia de movimento e de carga axial em maior quantidade aos discos adjacentes (hipersolicitação), que se tornam hipermóveis, com aumento da pressão intradiscal e alteração da cinemática do segmento, sob situação de hipersolicitação mecânica, como flexão-extensão, rotação e lateralização, em relação a discos em níveis equivalentes, resultando em degeneração em velocidade superior à observada espontaneamente.

Como alternativa à fusão e consequentes alterações biomecânicas nos segmentos adjacentes que resultam em sobrecarga e degeneração, a artroplastia cervical vem se popularizando sob a justificativa de preservação das condições biomecânicas do segmento, o que já foi demonstrado em caráter experimental e em estudos do tipo série de $\operatorname{casos}^{17,18}$.

\section{MÉTODOS}

Análise retrospectiva dos prontuários e exames de imagem (Radiografia simples neutra, em anteroposterior e lateral, Radiografia dinâmica em lateral, em flexão e extensão, Ressonância magnética e eventualmente a tomografia computadorizada da coluna cervical), de 209 casos de síndrome clínica de radiculopatia, mielopatia ou mielorradiculopatia submetidos a tratamento cirúrgico da doença degenerativa da coluna cervical. Utilizou-se a via anterior em 169 casos e a via posterior sem fusão em 40, no Hospital das Clínicas da Faculdade de Medicina da Universidade de São Paulo, no período de março de 1993 a janeiro de 2007.

$\mathrm{Na}$ via anterior, foi realizada a descompressão por discectomia em um ou múltiplos níveis ou a corpectomia. $\mathrm{Na}$ via posterior, utilizou-se a laminectomia sem fixação, a laminoplastia ou a arcocristectomia.

Excluíram-se os casos associados à artrite reumatoide, espondilite anquilosante, diabetes mellitus, alcoolismo, cirurgia prévia na coluna cervical, trauma cervical, seguimento clínico inferior a 24 meses e dados incompletos no prontuário.
A avaliação clínica foi embasada no exame neurológico no momento do diagnóstico com padrão de mielopatia ou radiculopatia e quantificada pela escala da JOA (Japanese Orthopaedic Association) traduzida (Tabela 1). Os pacientes foram reavaliados com base na mesma escala, do primeiro ao sétimo dia do pós-operatório (imediato) e na última avaliação ambulatorial, com um mínimo de 24 meses (tardia) quando utilizamos a taxa de recuperação (JOA) como referência (Tabela 2). Os pacientes foram distribuídos em grupos, de acordo com a via de acesso e a modalidade cirúrgica.

\section{TABELA 1 - Escala para mielopatia da Japanese Orthopaedic Association (JOA): 0 a 17 pontos}

\begin{tabular}{|c|c|}
\hline Impossível comer com colher ou abotoar camisa & 0 \\
\hline $\begin{array}{l}\text { Possível comer com colher, impossível abotoar } \\
\text { camisa }\end{array}$ & 1 \\
\hline Possível abotoar camisa, com grande dificuldade & 2 \\
\hline Possível abotoar camisa, com dificuldade & 3 \\
\hline Normal & 4 \\
\hline \multicolumn{2}{|l|}{ II - Função motora no membro inferior } \\
\hline Impossível & 0 \\
\hline Necessita de bengala ou auxílio em solo plano & 1 \\
\hline Necessita de auxílio em escadas & 2 \\
\hline Anda sem auxílio, mas lentamente & 3 \\
\hline Normal & 4 \\
\hline \multicolumn{2}{|l|}{ III - Função sensitiva } \\
\hline \multicolumn{2}{|l|}{ Membro superior } \\
\hline Distúrbio sensitivo aparente & 0 \\
\hline Distúrbio sensitivo mínimo & 1 \\
\hline Normal & 2 \\
\hline \multicolumn{2}{|l|}{ Membro inferior } \\
\hline Distúrbio sensitivo aparente & 0 \\
\hline Distúrbio sensitivo mínimo & 1 \\
\hline Normal & 2 \\
\hline \multicolumn{2}{|l|}{ Tronco } \\
\hline Distúrbio sensitivo aparente & 0 \\
\hline Distúrbio sensitivo mínimo & 1 \\
\hline Normal & 2 \\
\hline \multicolumn{2}{|l|}{ IV - Função vesical } \\
\hline Retenção urinária ou incontinência & 0 \\
\hline Sensação de retenção/perda de pequeno fluxo & 1 \\
\hline Retenção urinária e/ou polaciúria & 2 \\
\hline Normal & 3 \\
\hline
\end{tabular}


TABELA 2 - Taxa de recuperação (JOA)

Taxa de recuperação $(\%)=\frac{(\text { JOA Pós-op - JOA Pré-op }) \times 100}{17 \text { - JOA Pré-op }}$

A avaliação radiográfica consistiu na observação do alinhamento, presença ou não de lordose fisiológica, retificação ou inversão (cifotização) e anterolistese e retrolistese dos níveis adjacentes à fusão. A lordose cervical foi avaliada na radiografia em perfil neutro, traçando-se uma linha perpendicular ao platô inferior de $\mathrm{C} 2$ e platô superior de C7, medida ainda realizada nas incidências em perfil em flexão e extensão. Nas três situações, a medida entre cortical posteroinferior da vértebra superior à cortical posterosuperior da vértebra inferior dos níveis adjacentes à artrodese. Uma listese maior que $2 \mathrm{~mm}$ no neutro ou uma mudança maior que $2 \mathrm{~mm}$ nas distâncias dinâmicas definiram instabilidade ${ }^{19}$. Todos os pacientes com instabilidade no exame radiológico, estático ou dinâmico, foram submetidos à fixação interna com placa e parafusos.

A degeneração discal foi quantificada pela escala modificada de Kellgren (Tabela 3), nos períodos antes e 24 meses após a cirurgia em todos os grupos. Essa escala classifica progressivamente, na radiografia em perfil neutro, a severidade da degeneração discal, de zero ou ausente até 4 ou severa ${ }^{20}$.

\section{TABELA 3 - Escala modificada de Kellgren para doença degenerativa}

\begin{tabular}{ll}
\hline $\begin{array}{l}\text { Escore } \\
\text { Kellgren }\end{array}$ & Alterações radiográficas \\
\hline 0 & Sem alterações radiológicas \\
1 & Osteófito anterior \\
2 & Osteófito anterior, possível estenose, \\
& esclerose leve de platôs \\
3 & Osteófitos moderados, estenose, \\
& esclerose de platôs \\
4 & Osteófitos múltiplos, estenose severa, \\
& esclerose severa platôs
\end{tabular}

A ressonância magnética foi solicitada no pré-operatório em todos os casos, e novamente quando houve presença de novos sintomas clínicos ou nos casos sem melhora neurológica precoce após o tratamento cirúrgico. Tardiamente, foi solicitada em todos os casos. Onde havia evidências de calcificações ligamentares ou articulares, a tomografia computadorizada complementou o estudo radiológico para fins de diagnóstico acurado e planejamento cirúrgico.

\section{RESULTADOS}

A idade variou entre 30 e 84 anos, em 113 casos os pacientes eram do sexo masculino $(66,8 \%)$, em 56 , do sexo o feminino $(33,2 \%)$. O período decorrido entre o início dos sintomas e a cirurgia variou entre 1 e 72 meses. O período de seguimento pós-operatório variou entre 24 e 220 meses (Tabela 4).
TABELA 4 - Médias e percentuais de incidência das variáveis observadas na cirurgia pela via anterior

\begin{tabular}{|c|c|c|c|}
\hline & Geral & $\begin{array}{c}\text { Com } \\
\text { síndrome }\end{array}$ & $\begin{array}{c}\text { Sem } \\
\text { síndrome }\end{array}$ \\
\hline$f$ & 169 & 30 & 139 \\
\hline Idade & $50,57 \pm 11,84$ & $47,25 \pm 10,34$ & $51,62 \pm 12,09$ \\
\hline $\begin{array}{l}\text { Período de } \\
\text { evolução }\end{array}$ & $16,75 \pm 13,91$ & $14,91 \pm 11,75$ & $17,24 \pm 14,42$ \\
\hline $\begin{array}{l}\text { Seguimento } \\
\text { pós-operatório }\end{array}$ & $54,91 \pm 35,37$ & $75,82 \pm 45,95$ & $48 \pm 28,12$ \\
\hline Mielopatia & $111(65,68)$ & $20(66,6 \%)$ & $91(65,46 \%)$ \\
\hline Radiculopatia & $9(53,25 \%)$ & $5(16,6 \%)$ & $4(2,87 \%)$ \\
\hline Mielorradiculopatia & 49 (28,99\%) & $5(16,6 \%)$ & $44(31,65 \%)$ \\
\hline Dor cervical & 112 (63,3\%) & $25(83,3 \%)$ & $87(62,58 \%)$ \\
\hline Hoffman & 145 (85\%) & $26(86,6 \%)$ & $119(85,61 \%)$ \\
\hline \multicolumn{4}{|l|}{$\begin{array}{l}\text { Instabilidade } \\
\text { radiológica }\end{array}$} \\
\hline Estática & $20(11,83 \%)$ & $1(3,3 \%)$ & $19(13,66 \%)$ \\
\hline $\begin{array}{l}\text { Estática/ } \\
\text { Dinâmica }\end{array}$ & $2(1,18 \%)$ & 0 & $2(1,43 \%)$ \\
\hline Dinâmica & $4(2,36 \%)$ & $1(3,3 \%)$ & $3(2,15 \%)$ \\
\hline $\begin{array}{l}\text { Inversão da } \\
\text { lordose }\end{array}$ & $40(23,66 \%)$ & $11(36,6 \%)$ & 29 (20,86\%) \\
\hline $\begin{array}{l}\text { Alteração de } \\
\text { curvatura }\end{array}$ & $72(42,60 \%)$ & 15 (50\%) & 57 (41\%) \\
\hline $\begin{array}{l}\text { Compressão em } \\
\text { múltiplos níveis }\end{array}$ & $101(59,76 \%)$ & 12 (40\%) & 89 (64\%) \\
\hline $\begin{array}{l}\text { Cirurgia em } \\
\text { múltiplos níveis }\end{array}$ & $45(26,62 \%)$ & $2(6,6 \%)$ & 43 (30,93\%) \\
\hline
\end{tabular}

O período decorrido entre a cirurgia e o início de novos sintomas (mielopatia ou radiculopatia) foi de $28,45 \pm 30,92$ meses.

A média da pontuação e a taxa de recuperação, segundo a escala da JOA, apresentadas na Tabela 5, evidenciam a progressão da evolução clínica entre os períodos antes e após a cirurgia, quantificados em pontos e variando entre zero e 17. Foram utilizadas médias dos grupos sem e com síndrome e da população geral.

TABELA 5 - Média da pontuação na escala da JOA com e sem a síndrome do disco adjacente e da taxa de recuperação via anterior

\begin{tabular}{lccc}
\hline & Geral & $\begin{array}{c}\text { Sem } \\
\text { síndrome }\end{array}$ & $\begin{array}{c}\text { Com } \\
\text { síndrome }\end{array}$ \\
\hline $\mathbf{f}$ & $\mathbf{n}=169$ & $\mathbf{n}=139$ & $\mathbf{n}=30$ \\
\hline JOA Pré & $9,55 \pm 2,51$ & $9,38 \pm 2,52$ & $10,11 \pm 2,39$ \\
JOA Pós & $12,78 \pm 2,54$ & $12,54 \pm 2,53$ & $13,58 \pm 2,45$ \\
JOA Tardio & $12,66 \pm 2,66$ & $12,68 \pm 2,65$ & $12,51 \pm 2,72$ \\
Taxa de & $42,79 \pm 28,2$ & $40,78 \pm 27,07$ & $49,96 \pm 31,26$ \\
recuperação & & & \\
precoce & & & \\
$\begin{array}{l}\text { Taxa de } \\
\text { recuperação } \\
\text { tardia }\end{array}$ & $41,40 \pm 31,36$ & $43,19 \pm 29,28$ & $35,04 \pm 37,67$ \\
\hline
\end{tabular}


A melhora pós-operatória mostrou-se significativa $(\mathrm{p}=0,001)$ independentemente da ocorrência da síndrome na via anterior. Também houve melhora significativa nos casos de cirurgia pela via posterior, de acordo com a Tabela 6.

TABELA 6 - Média da pontuação na escala da JOA e da taxa de recuperação vias anterior e posterior

\begin{tabular}{lcc}
\hline & Anterior & Posterior \\
\hline $\mathrm{f}$ & $\mathrm{n}=169$ & $\mathrm{n}=40$ \\
\hline JOA Pré & $9,55 \pm 2,51$ & $9,95 \pm 2,38$ \\
JOA Pós & $12,78 \pm 2,54$ & $11,77 \pm 2,93$ \\
JOA Tardio & $12,66 \pm 2,66$ & $11,8 \pm 3,10$ \\
Taxa de recuperação precoce & $42,79 \pm 28,2$ & $27,92 \pm 24,75$ \\
Taxa de recuperação tardia & $41,40 \pm 31,36$ & $26,92 \pm 31,84$ \\
\hline
\end{tabular}

O resultado final do tratamento via anterior é apresentado na Tabela 7.

Em 70 casos, houve diagnóstico de novas degenerações ou progressão de degenerações pré-existentes, avaliados pela escala modificada de Kellgren (Tabela 8).

TABELA 7 - Resultado final nos períodos
precoce a tardio após tratamento pela via
anterior
\begin{tabular}{lcccc}
\hline & $f$ & Melhora & Inalterado & Piora \\
\hline Imediato & 169 & $145(85,8 \%)$ & $16(9,5 \%)$ & $8(4,7 \%)$ \\
Tardio & 169 & $139(82,2 \%)$ & $20(11,8 \%)$ & $10(6 \%)$ \\
\hline
\end{tabular}

TABELA 8 - Escala de Kellgren pré-operatório e pós-operatório para os níveis adjacentes, via anterior

\begin{tabular}{|c|c|c|c|c|}
\hline \multirow[b]{2}{*}{$\begin{array}{l}\text { Escore de } \\
\text { Kellgren }\end{array}$} & \multicolumn{2}{|c|}{ Pré-operatório } & \multicolumn{2}{|c|}{ Pós-operatório } \\
\hline & $\begin{array}{c}\text { Com } \\
\text { síndrome }\end{array}$ & $\begin{array}{c}\text { Sem } \\
\text { síndrome }\end{array}$ & $\begin{array}{c}\text { Com } \\
\text { síndrome }\end{array}$ & $\begin{array}{c}\text { Sem } \\
\text { síndrome }\end{array}$ \\
\hline & n (\%) & n (\%) & n (\%) & n (\%) \\
\hline 0 & $9(30)$ & $59(42,4)$ & 0 & $33(23,7)$ \\
\hline 1 & $6(20)$ & $43(30,9)$ & $6(20)$ & $49(35,2)$ \\
\hline 2 & $6(20)$ & $31(22,3)$ & $11(33,6)$ & $41(29,5)$ \\
\hline 3 & $9(30)$ & $6(4,31)$ & $13(43,3)$ & $16(11,5)$ \\
\hline 3 & $9(30)$ & $6(4,31)$ & $13(43,3)$ & $16(11,5)$ \\
\hline 4 & 0 & 0 & 0 & 0 \\
\hline
\end{tabular}

No total, houve um aumento de $41,42 \%$ das alterações radiológicas degenerativas nos discos adjacentes à fusão após a cirurgia. Não houve correlação desta piora radiológica com o desenvolvimento de nova sintomatologia clínica após a fusão intersomática.

Nos 40 casos em que foi utilizada a via posterior, houve progressão em 21 casos $(52,5 \%)$, dos quais somente 2 apresentaram novo quadro clínico relacionado à instabilidade e sem compressões presentes no período pré-operatório.

No grupo com síndrome clínica após a fusão (30 casos), houve correlação do nível das compressões diagnosticadas com alterações degenerativas discais pré-existentes leves a moderadas em 21 casos $(70 \%)$, diferença estatisticamente significativa para $\mathrm{p}=0,001$.

A frequência de alterações no alinhamento sagital no grupo da síndrome foi de $50 \%$, sendo que no grupo sem a síndrome foi de $41 \%$. A frequência de inversão da lordose no grupo da síndrome foi de $36,6 \%$ e de $20,86 \%$ no grupo sem a síndrome.

Foi diagnosticada a síndrome do disco adjacente em 30 pacientes $(17,75 \%)$ operados pela via anterior, como apresentado na Tabela 9.

\begin{tabular}{lcc}
\multicolumn{3}{l}{ TABELA 9 - Número de casos e discos } \\
\multicolumn{4}{c}{ acometidos pela Síndrome do disco adjacente } \\
\hline Superior & Casos & Discos \\
Inferior & $3+(10)$ & $8+(10)$ \\
Ambos & $7+(10)$ & $13+(10)$ \\
Total & -10 & -10 \\
\hline
\end{tabular}

Na Tabela 10, os casos foram distribuídos de acordo com a modalidade cirúrgica e com a ocorrência da síndrome de transição.

Houve efetivação de reintervenção cirúrgica em 19 casos $(63,3 \%)$. No total, 11 pacientes $(36,6 \%)$ foram tratados clinicamente com sucesso.

Os sítios da cirurgia primária nos casos de síndrome estão expostos na Tabela 11.

Em dois casos, a cirurgia primária foi em níveis múltiplos, um sem fixação e um com fixação.

\section{DISCUSSÃO}

Evidências biomecânicas de sobrecarga em discos adjacentes à fusão no segmento subaxial da coluna cervical foram demonstradas em estudos como os de Dimitriev ${ }^{18}$, Hilibrand $^{21}$, Eck $^{22}$, Maiman ${ }^{23}$, Lopez-Espina ${ }^{24}$.

Em seu estudo biomecânico utilizando espécimes cadavéricos de coluna cervical de humanos, Eck demonstrou significatico aumento na pressão intradiscal e amplitude de movimento em segmentos adjacentes à fusão em situações normais de amplitude de movimento em flexão, extensão e rotação, concluindo que este fenômeno poderia explicar parcialmente a degeneração precoce dos discos em segmentos adjacentes a áreas de fusão. Lopez-Espina denotou 96\% de aumento de estresse mecânico no núcleo, ânulo e placa terminal em níveis adjacentes a segmentos cervicais fundidos, utilizando um modelo cadavérico. Aumento no estresse mecânico foi notado seguindo-se uma fusão em dois níveis em relação a nível único. 


\begin{tabular}{|c|c|c|c|}
\hline $\begin{array}{l}\text { Modalidade } \\
\text { cirúrgica }\end{array}$ & $\mathrm{n}$ & $\begin{array}{l}\text { Síndrome do } \\
\text { disco adjacente }\end{array}$ & $\%$ relativa \\
\hline $\begin{array}{l}\text { Discectomia sem } \\
\text { indução/fusão }\end{array}$ & 60 & 12 & 20 \\
\hline $\ln$ & 43 & 9 & 20,9 \\
\hline $2 n$ & 16 & 3 & 18,7 \\
\hline $3 n$ & 1 & 0 & - \\
\hline Acrílico & 9 & 0 & - \\
\hline Nada & 51 & 12 & 23,52 \\
\hline $\begin{array}{l}\text { Discectomia com } \\
\text { fusão sem fixação }\end{array}$ & 44 & 8 & 18 \\
\hline Cage & 32 & 5 & 15,6 \\
\hline llíaco & 12 & 3 & 25 \\
\hline $\ln$ & 32 & 7 & 21,8 \\
\hline $2 n$ & 10 & 1 & 10 \\
\hline $3 n$ & 2 & 0 & - \\
\hline $\begin{array}{l}\text { Discectomia com } \\
\text { fusão com fixação }\end{array}$ & 31 & 10 & 32,2 \\
\hline Cage/Placa & 9 & 2 & 22,2 \\
\hline Ilíaco/Placa & 0 & 0 & - \\
\hline $\begin{array}{l}\text { Cage-Placa } \\
\text { conjugados }\end{array}$ & 22 & 8 & 36,36 \\
\hline $\ln$ & 24 & 9 & 37,5 \\
\hline $2 n$ & 6 & 1 & $16,60 \%$ \\
\hline $3 n$ & 1 & 0 & - \\
\hline Corpectomia & 34 & 0 & - \\
\hline $\ln$ & 25 & 0 & - \\
\hline $2 n$ & 7 & 0 & - \\
\hline $3 n$ & 2 & 0 & - \\
\hline
\end{tabular}

TABELA 11 - Níveis primários da cirurgia

\begin{tabular}{lcc}
\hline Nível & $f$ & $\%$ \\
\hline C3-C4 & 5 & 16,6 \\
C4-C5 & 4 & 13,3 \\
C5-C6 & 17 & 56,6 \\
C6-C7 & 4 & 13,3 \\
C4-C5/C6-C7 & 1 & 3,3 \\
C3-C4/C5-C6 & 1 & 3,3 \\
\hline
\end{tabular}

Em outro estudo experimental com modelo cadavérico de coluna cervical de humanos, Dimitriev comparou espécimes intactos e reconstruídos no nível C5-C6 com: (1) prótese discal total, (2) espaçador intersomático e (3) espaçador intersomático + placa cervical anterior. Testes foram executados com controle de deslocamento em rotação axial, flexão/extensão, e lateralização. Medidas de pressão intradiscal (PID) foram colhidas nos níveis C4-C5 e C6-C7, enquanto o pico de alcance de movimento (PAM) foi monitorizado no nível C5-C6. PIDs similares foram encontrados entre a condição intacta e a reconstrução com prótese de disco total em ambos os níveis adjacentes e sob todos os modelos de carga ( $>00,05)$. Entretanto, os valores de PID para o nível C4-C5 produzidos sob o teste de flexão/extensão para ambos os modelos de artrodese foram significativamente maiores do que as médias obtidas para o modelo intacto e prótese discal $(p<0,05)$. Diferenças similares intergrupos foram observadas no nível C6-C7, porém significância estatística foi obtida durante todos os três métodos de carga $(\mathrm{p}<0,05)$. A análise do nível C5C6 indicou um significatico PAM menor para ambas as construções de artrodese em comparação ao grupo intacto e prótese de disco total durante o teste de flexão/extensão $(\mathrm{p}<0,05)$. Nenhuma diferença foi registrada entre $o$ grupo intacto e prótese discal total em condição de carga $(\mathrm{p}>0,05)$. O estudo documentou que a prótese discal total cervical mantém as medidas de pressão intradiscal e a cinemática da coluna nos níveis adjacentes próximas aos valores pré-operatórios, o que não se aplica ao caso das fusões, podendo fornecer uma alternativa no manejo da cirurgia convencional para a doença discogênica cervical.

Entretanto, o momento em que esse aumento de estresse de movimento em discos adjacentes a segmentos de artrodese se torna um problema clínico relevante é desconhecido.

Katsuura $^{25}$ observou evidentes alterações degenerativas nos exames radiológicos nos níveis adjacentes a um segmento fundido em 21 de 42 pacientes (50\%), após fusão cervical intersomática por via anterior, com média de seguimento de 9,8 anos. Goffin ${ }^{26}$ relatou resultados de 181 pacientes tratados com fusão intersomática cervical via anterior, com média de segmento de 8 anos, relatando suave deterioração radiológica em níveis adjacentes em 92\% dos casos estudados e deterioração moderada a grave em $43 \%$ dos casos. A severidade das alterações radiológicas foi associada ao intervalo de tempo transcorrido desde a cirurgia, mas a degeneração discal radiológica em nível adjacente não foi correlacionada com sintomas clínicos.

Herkowits $^{27}$ estudou prospectivamente 44 pacientes com radiculopatia cervical, randomizados para uma via anterior com discectomia e fusão ou uma via posterior com foraminotomia, com média de seguimento pós-procedimento de 4,5 anos. Entre os submetidos à fusão por via anterior, $41 \%$ desenvolveram degeneração adjacente radiográfica, enquanto por via posterior com foraminotomia sem fusão, $50 \%$ desenvolveram degenerações discais radiológicas adjacentes. Como no estudo citado anteriormente, não foi relatada correlação entre achados radiológicos e sintomas clínicos. Diversos investigadores vêm tentando avaliar a prevalência da degeneração do disco adjacente à fusão com alterações clínicas significativas, entre eles Hilibrand ${ }^{14}$ que, em um estudo com longo seguimento clínico, demonstrou que mais de um quarto dos pacientes é acometido por novo quadro de radiculopatia ou mielopatia 
relacionado a um segmento adjacente imóvel dentro de 10 anos após artrodese cervical. $\mathrm{O}$ estudo demonstrou uma incidência anual de aproximadamente $2,9 \%$ de doença sintomática após o procedimento. Uma artrodese em um nível envolvendo a quinta ou a sexta vértebra cervical e havendo pré-existência da evidência radiográfica de degeneração de disco adjacente se mostra um grande fator de risco para o surgimento de nova doença. Surpreendentemente, o estudo mostra que o risco para uma nova doença em um disco adjacente à fusão é significativamente menor seguido a uma artrodese em múltiplos níveis em uma artrodese em um único nível. A hipótese é de que este achado pode ter sido devido à inclusão de segmentos móveis de alto risco nos procedimentos.

Yue $^{28}$ avaliou resultados clínicos e radiológicos por um período médio de 7,2 anos após discectomia e fusão cervical via anterior. Nesta série, 12 de 71 pacientes $(16,9 \%)$ necessitaram de um segundo procedimento para tratamento de um disco adjacente sintomático, com média de 41,8 meses após o procedimento inicial. Em um estudo similar, Ishihara ${ }^{29}$ determinou uma incidência de doença discal adjacente sintomática de 19\% entre 112 pacientes estudados. Os sintomas se desenvolveram com uma media de 6,5 anos após o procedimento inicial. Entre os pacientes que desenvolveram doença discal adjacente sintomática, $37 \%$ não obtiveram sucesso em um tratamento conservador e foi necessário novo procedimento. $\mathrm{O}$ cálculo do índice de sobrevida de Kaplan-Meier determinou uma sobrevida livre de doença de $89 \%$ após 5 anos, $84 \%$ após 10 anos e $67 \%$ após 17 anos. Protrusão discal no nível adjacente presente na mielografia ou na imagem da ressonância magnética (RNM) pré-operatória foi associada ao desenvolvimento da doença do disco adjacente. Com base neste estudo, a incidência de degeneração discal em nível adjacente, radiográfica, é estimada entre 5 e $9 \%$ ao ano. Entretanto, doença do disco adjacente sintomática aparentemente ocorre em 2 a 3\% ao ano, após discectomia e fusão por via anterior. Em estudos prévios, a incidência de doença sintomática girou em torno de 7 e $15 \%{ }^{14}$. A identificação dos fatores correlacionados ao desenvolvimento da doença em 112 pacientes, acompanhados segundo critérios clínicos e radiológicos por mais de 2 anos, mostrou em 19 casos novo quadro de doença discal sintomática (19\%). A análise de sobrevida de Kaplan-Meier evidenciou taxas de sobrevida livre de doença de $89 \%$ aos 5 anos, $84 \%$ aos 10 anos e $67 \%$ aos 17 anos. A incidência de protrusão discal na RNM pré-operatória foi significativamente maior nos casos de doença ( $\mathrm{p}=0,0087 \mathrm{e} \mathrm{p}=0,0299$, respectivamente, Teste do $\chi^{2}$ ). Entretanto, os outros parâmetros não evidenciaram diferenças estatisticamente significativas. Em sete casos (37\%), houve necessidade de reintervenção cirúrgica por falha no tratamento conservador. A incidência da doença discal adjacente sintomática é maior quando a RNM pré-operatória evidencia degeneração discal adjacente assintomática em detrimento dos demais parâmetros, como número de níveis fundidos, alinhamento pré-operatório, diâmetro do canal ou alinhamento sagital.
Na presente série de 160 casos abordados pela via anterior e com fusão, com média de seguimento de $54,91 \pm 35,37$ meses, observou-se a ocorrência da síndrome do disco adjacente em 30 pacientes (17,75\%), com uma média de 2,9 casos/ano e média de surgimento de novos sintomas de $28,5 \pm 30,92$ meses. Resultados descritos na literatura para taxas de ocorrência da síndrome variam entre 7 e $50 \%$, mais comumente em torno de $15 \%$, tendo sido descrita por Hilibrand ${ }^{14}$ uma taxa anual de ocorrência de 2,9 casos/ano.

Nos casos abordados por via posterior sem fusão $(n=40)$ e nos casos em que houve interposição de material acrílico no espaço do disco removido $(\mathrm{n}=9)$, observaramse, após a cirurgia, dois novos casos de mielorradiculopatia relacionados a herniações em segmentos que se tornaram instáveis.

A melhora clínica dos pacientes submetidos à descompressão por via anterior foi verificada entre o primeiro e o sétimo dia após a cirurgia, com uma ligeira queda tardia evidenciada pelo índice de recuperação precoce e tardio, com diferenças estatisticamente significativas para $\mathrm{p}=000,1$. A ligeira queda tardia no índice não mostra significância estatística $(\mathrm{p}=0,005)$. No grupo da síndrome $(n=30)$, os escores indicam piora tardia mais notável (1 ponto) e maior variância no escore de recuperação tardio em relação ao grupo sem a síndrome, no qual a piora tardia é mínima, mostrando interferência negativa da síndrome do disco adjacente na evolução clínica, porém sem significância estatística. Nos 40 pacientes do grupo submetido à cirurgia por via posterior, os escores evidenciam melhora precoce e tardiamente.

A ocorrência da síndrome, em média, foi evidenciada após $28,45 \pm 30,92$ meses após a cirurgia.

Com a análise dos exames radiológicos, observamos a progressão ou o surgimento de novas e evidentes alterações degenerativas nos níveis adjacentes a um segmento fundido, por via anterior, em 70 casos $(41,42 \%)$ ou uma taxa de degeneração de $6,7 \%$ ao ano. Essas alterações não foram correlacionadas com sintomas clínicos. Quando analisados os 30 casos em que ocorreu o surgimento de novo quadro clínico, foram observadas alterações radiológicas pré-fusão em 22 pacientes (70\%) e de maior gravidade em comparação às alterações discais pré-fusão do grupo sem a síndrome. No grupo de pacientes submetidos à cirurgia por via posterior, houve progressão ou surgimento de novas alterações degenerativas em segmentos adjacentes em $52,5 \%$ dos casos, também sem correlação com alterações clínicas observadas em 5\% dos casos.

A cirurgia primária no nível C5-C6 demonstrou ser um fator associado ao risco de ocorrência da síndrome com $56,7 \%$ dos casos associados a este nível contra $13,3 \%$ nos níveis C4-C5 e C6-C7, e 16,7\% no nível C3-C4, sendo o risco inversamente proporcional ao número de segmentos fundidos, resultado similar ao demonstrado por Hilibrand ${ }^{13,14}$. $\mathrm{O}$ disco inferior foi mais frequentemente acometido.

Um novo procedimento para descompressão foi efetivado em 19 casos $(63,3 \%)$ e em 11 casos o tratamento conservador obteve êxito. 
Alterações no alinhamento sagital nos exames radiográficos em perfil (inversão ou retificação da lordose) foram observadas em 50\% dos casos com síndrome, dados semelhantes ao da literatura, contra $41 \%$ no grupo sem, a síndrome com menor gravidade.

Segundo os critérios de instabilidade ${ }^{19}, 20$ casos $(11,83 \%)$ apresentavam listese na radiografia estática, 2 deles $(1,18 \%)$ com piora da instabilidade na radiografia dinâmica, e em 4 casos $(2,36 \%)$ a instabilidade somente foi diagnosticada pela radiografia dinâmica, sem evidência na radiografia em perfil estático.

Quanto à modalidade cirúrgica, a ocorrência da síndrome de transição foi significativamente maior nos casos em que a fusão foi associada à fixação com placas ou espaçador intersomático conjugado à placa, nos casos de fusão em único nível, não sendo identificado caso associado à fusão em três níveis, em casos de corpectomia, em casos de discectomia com interposição de acrílico e em casos onde ocorreu pseudoartrose (Tabela 10), corroborando para a associação da fusão com o surgimento de novo quadro clínico.

\section{CONCLUSÕES}

Observou-se a ocorrência de altas taxas de fusão e alguns casos de pseudoartrose em proporções semelhantes em todos os grupos.
A frequência e a gravidade das alterações no alinhamento sagital da coluna cervical no grupo com a síndrome foi superior ao observado no grupo sem a síndrome.

A degeneração discal progressiva foi observada no período pós-operatório dos grupos em que foi realizada a via anterior ou posterior, com incidência semelhante $\mathrm{e}$ sem relação com o quadro clínico. Entretanto, nos casos em que a descompressão e a fusão foram feitas por via anterior, constatou-se o desenvolvimento da síndrome do disco adjacente, com acometimento por novo quadro clínico de mielorradiculopatia no período pós-cirúrgico. Verificou-se o início da manifestação clínica após dois anos de evolução pós-operatória e uma tendência cronológica progressiva ao desenvolvimento, causando deterioração neurológica tardia.

A verificação de alteração discal nos exames radiológicos pré-existentes à fusão em níveis adjacentes, assim como fusões em um único nível e associação à fusão com placas ou uma fusão que envolva a quinta ou a sexta vértebra cervical, são fatores predisponentes ao desenvolvimento da síndrome.

Não foi observada a síndrome do disco adjacente nos casos em que não ocorreu a fusão (pseudoartrose) e nos casos em que houve interposição de metacrilato no espaço da discectomia ou na via posterior sem fusão.

\section{REFERÊNCIAS}

1. Spurling RG, Scoville WB. Lateral rupture of the cervical intervertebral discs. A common cause of shoulder and arm pain. Surg Gynecol Obstet. 1944;78(4):350-8.

2. Cloward RB. The anterior approach for removal of ruptured cervical discs. J Neurosurg. 1958;15(6):602-17.

3. Smith GW, Robinson RA. The treatment of certain cervical spine disorders by anterior removal of the intervertebral disc and interbody fusion. J Bone Joint Surg. 1958;40(3):607-24.

4. Smith GW, Robinson RA. Anterolateral cervical disc removal and interbody fusion cervical disc syndrome. Bull John Hopkins Hosp. 1955;96(Suppl):223-4.

5. Chesnut RM, Abitbol JJ, Garfin SR. Surgical management of cervical radiculopathy. Indication, techniques, and results. Orthop Clin North Am. 1992;23(3):461-74.

6. Mosdal C. Cervical osteochondrosis and disc herniation. Eighteen years' use of interbody fusion by Cloward's technique in 755 cases. Acta Neurochir. 1984;70(3-4):207-25.
7. Samii M, Volkening D, Sepehrnia A, Penkert G, Baumann H. Surgical treatment of myeloradiculopathy in cervical spondylosis. A report on 438 operations. Neurosurg Rev. 1989;12(4):285-90.

8. Scoville WB, Dohrmann GJ, Corkill G. Late results of cervical disc surgery. J Neurosurg. 1976;45(2):203-10.

9. Viejo-Fuertes D, Liguoro D, Ansari M, Rombouts JJ, Vital JM, Sénégas J. Complications following anterior approaches to the cervical spine. Review of 535 surgical procedures. Eur J Orthop Surg Traumatol. 2000;10(3):177-81.

10.Levin DA, Hale JJ, Bendo JA. Bulletin of the NYU Hospital for Joint Diseases. Adjacent segment degeneration following spinal fusion for degenerative disc disease. Bull NYU Hosp Jt Dis. 2007;65(1):29-36.

11.Kawakami M, Tamaki T, Ando M, Yamada H, Hashizume H, Yoshida M. Lumbar sagittal balance influences the clinical outcome after decompression and posterolateral spinal fusion for degenerative lumbar spondylolisthesis. Spine. 2002;27(1):59-64.
12.Lagrone MO, Bradford DS, Moe JH, Lonstein JE, Winter RB, Ogilvie JW. Treatment of symptomatic flatback after spinal fusion. J Bone Joint Surg Am. 1988;70(4):569-80.

13. Hilibrand AS, Carlson GD, Palumbo MA, Jones PK, Bohlman HH. Radiculopathy and myelopathy at segments adjacent to the site of a previous anterior cervical arthrodesis. J Bone Joint Surg Am. 1999;81(4):519-28.

14.Hilibrand AS, Robbins M. Adjacent segment degeneration and adjacent segment disease: the consequences of spinal fusion? Spine J. 2004;4(6 Suppl):190S-194S.

15.Gore DR, Sepic SB. Anterior cervical fusion for degeneration or protruded discs. A review of one hundred fortysix patients. Spine. 1984;9(7):667-71.

16. Buckwalter JA. Aging and degeneration of the human intervertebral disc. Spine. 1995;20(11):1307-14.

17.Dahl MC, Rouleau JP, Papadopoulos S, Nuckley DJ, Ching RP. Dynamic characteristics of the intact, fused and prosthetic-replaced cervical disk. J Biomecch Eng. 2006;128(6):809-14. 
18. Dmitriev AE, Cunningham BW, Hu N, Sell G, Vigna F, McAfee PC. Adjacent level intradiscal pressure and segmental kinematics following a cervical total disc arthroplasty: an in vitro human cadaveric model. Spine (Phila Pa 1976). 2005;30(10):1165-72.

19. White AP, Biswas D, Smart LR, Haims A, Grauer JN. Utility of flexion-extension radiographs in evaluating the degenerative cervical spine. Spine. 2007;32(9):975-9.

20.Kellgren JH, Jeffrey MR, Ball J, editors. The epidemiology of chronic rheumatism. Atlas of standard radiographs. Oxford: Blackwell Scientific; 1963. p. 14-9.

21.Hilibrand AS, Balasubramanian K, Eichenbaum M, Thinnes JH, Daffner $\mathrm{S}$, Berta S, et al. The effect of anterior cervical fusion on neck motion. Spine. 2006;31(15):1688-92.

22.Eck JC, Humphreys SC, Lim TH, Jeong ST, Kim JG, Hodges SD, et al. Biomechanical study on the effect of cervical spine fusion on adjacent-level intradiscal pressure and segmental motion. Spine. 2002;27(22):2431-4.
23.Maiman DJ, Kumaresan S, Yoganandan N, Pintar FA. Biomechanical effect of anterior cervical spine fusion on adjacent segments. Biomed Mater Eng. 1999;9(1):27-38.

24.Lopez-Espina CG, Amirouche F, Havalad V. Multilevel cervical fusion and its effect on disc degeneration and osteophyte formation. Spine. 2006;31(9):972-8.

25.Katsuura A, Hukuda S, Saruhashi Y, Mori K. Kyphotic malalignment after anterior cervical fusion is one of the factors promoting the degenerative process in adjacent intervertebral levels. Eur Spine J. 2001;10(4):320-4.

26.Goffin J, Geusens E, Vantomme N, Quintens E, Waerzeggers Y, Depreitere B, et al. Long-term follow-up after interbody fusion of the cervical spine. J Spinal Disord. 2004;17(2):79-85.

27.Herkowitz HN, Kurz LT, Overholt DP. Surgical management of cervical soft disc herniation. A comparison between the anterior and posterior approach. Spine. 1990;15(10):1026-30.
28. Yue WM, Brodner W, Highland TR. Long-term results after anterior cervical discectomy and fusion with allograft and plating. A 5- to 11-year radiologic and clinical follow-up study. Spine. 2005;30(19):2138-44.

29.Ishihara H, Kanamori M, Kawaguchi Y, Nakamura H, Kimura T. Adjacent segment disease after anterior cervical interbody fusion. Spine J. 2004;4(6):624-8.

\section{Correspondência}

Av. Dr. Enéas de Carvalho Aguiar, 255

CEP 05403-000 - São Paulo (SP), Brasil

Fone: (1 1 ) 3069-6000

E-mail: alemeluzzi@ig.com.br 